\title{
Shifting the paradigm for maintenance therapy in advanced non-small-cell lung cancer
}

This article was published in the following Dove Press journal:

Lung Cancer:Targets and Therapy

5 June 2010

Number of times this article has been viewed

\author{
Barbara Melosky \\ University of British Columbia, \\ Vancouver, BC, Canada
}

Correspondence: Barbara Melosky $600 \mathrm{~W}$ I0th Ave Vancouver,

BCV5Z 4E6, Canada

Tel + I 604-877-6000

$\mathrm{Fax}+$ I 604-875-0585

Email bmelosky@bccancer.bc.ca

\begin{abstract}
The standard of care in metastatic non-small-cell lung cancer (NSCLC) is to treat patients with a platinum doublet for 4 to 6 cycles and to offer second-line therapy upon progression. Maintenance therapy in NSCLC is defined as a therapeutic agent which is administered after first-line chemotherapy is complete, but before the disease progresses. While maintenance therapy is not yet universally accepted as a therapeutic approach, emerging data have demonstrated its potential to improve progression-free survival and overall survival in a clinically significant way. In this article a concise recent review of data related to the role of maintenance therapy in patients with advanced NSCLC is provided, complementing previous information in the field. It is recommended that NSCLC patients are offered maintenance therapy after first-line therapy is complete.
\end{abstract}

Keywords: advanced non-small-cell lung cancer, maintenance therapy, pemetrexed, erlotinib

Standard therapy for advanced stage non-small-cell lung cancer (NSCLC) includes first-line chemotherapy with a platinum-based doublet. ${ }^{1}$ Patients are treated with four to six treatment cycles, with the vast majority receiving four treatment cycles. Maintenance therapy in NSCLC refers to treatment given after first-line chemotherapy, and before disease progression. Although early trials suggested that prolonged or maintenance chemotherapy is not beneficial, ${ }^{2,3}$ new evidence reveals an overall survival (OS) advantage for both maintenance pemetrexed and erlotinib. Both of these therapies are currently approved and used upon progression following front-line chemotherapy; however new evidence demonstrates superior results when these therapies are used before progression occurs.

The use of pemetrexed in a maintenance setting was presented at ASCO 2009 by Ciuleanu et al and published in the September 20, 2009 issue of the Lancet. ${ }^{4}$ A total of 663 patients with Stage IIIB/IV NSCLC who had not progressed after four cycles of platinum-based chemotherapy were randomly assigned (2:1 ratio) to receive maintenance pemetrexed $500 \mathrm{mg} / \mathrm{m}^{2}$ plus best supportive care $(\mathrm{n}=441)$ or placebo plus best supportive care $(n=222)$. Both primary and secondary endpoints were significantly in favor of the pemetrexed arm. The primary endpoint of progression-free survival (PFS) was 4.3 months versus 2.6 months $(P<0.0001$ ), hazard ratio (HR) 0.50 in favor of maintenance pemetrexed. The secondary endpoint of OS was 13.4 months versus 10.6 months $(P=0.012)$, HR 0.79 in favor of maintenance pemetrexed. The results are even more impressive in the non-squamous subgroup where PFS HR 0.44 $(P<0.001$ and OS HR $0.70(P=0.002)))^{5,6}$ submit your manuscript | www.dovepress.com which permits unrestricted noncommercial use, provided the original work is properly cited. 
The influence of erlotinib, an oral tyrosine kinase inhibitor (TKI), in the epidermal growth factor receptor (EGFR) mutation and wild-type tumors was studied in a maintenance fashion in trials SATURN and ATLAS. In SATURN, the use of maintenance erlotinib after a platinum-based doublet was tested in a randomized placebo controlled fashion. ${ }^{7}$ Almost 2000 patients received first-line therapy, of which 889 patients did not experience disease progression. The nonprogressing patients were randomized to erlotinib $150 \mathrm{mg}$ daily or placebo. Both coprimary endpoints, PFS in all patients, and PFS in patients with EGFR IHC+ tumors, were positive. The overall PFS HR was 0.71 (95\% CI, 0.62-0.82; $P<0.0001)$. The coprimary endpoint assessed a subgroup of patients with tumors positive for EGFR protein expression determined by immunohistochemistry. The PFS HR in this group was 0.69 (95\% CI, $0.58-0.92 ; P<0.0001)$ favoring erlotinib. There was an OS benefit of 1 month: 12 months versus 11 months ( $\mathrm{HR}=0.81 \log$-rank $P=0.0088)$. In the non-squamous group, OS was greater than 3 months, with 13.7 month survival versus 10.5 month survival (HR 0.79 $\log$-rank $P=0.0194$ ).

The ATLAS study evaluated a maintenance combination therapy of EGFR TKI erlotinib with the vascular endothelial growth factor (VEGF) inhibitor bevacizumab. ${ }^{8}$ Patients who were treated with bevacizumab in addition to platinumcontaining doublet therapy and who had not progressed, were randomized to erlotinib or placebo for maintenance therapy. ${ }^{8}$ The trial was stopped by the data safety monitoring committee at the second interim evaluation because the primary endpoint of PFS was met. The absolute improvement in PFS was approximately 1 month, 4.8 months versus 3.7 months (HR $0.72 ; 95 \%$ CI, $0.59-0.81 ; P=0.0012$ ). While OS data are pending, the ATLAS trial supports the use of erlotinib for maintenance therapy.

A recent meta-analysis of 13 maintenance chemotherapy trials demonstrated an improvement in PFS and in OS. ${ }^{9}$ The most promising strategies involved administering an approved second-line NSCLC therapeutic for maintenance therapy. ${ }^{4,10}$

Thus, emerging data have demonstrated that maintenance therapy statistically improves survival in advanced NSCLC. Both pemetrexed and erlotinib have been found to improve both PFS and OS when administered as maintenance therapy after first-line chemotherapy is completed. Administering second-line maintenance therapies immediately following first-line chemotherapy before progression, is a useful strategy to enhance PFS. This represents a paradigm shift in the treatment of this malignancy.

\section{Background}

Chemotherapy with a platinum doublet for 4 to 6 cycles is considered the standard of care for first-line treatment in eligible patients with metastatic NSCLC. Second-line therapy is given upon disease progression and options include pemetrexed ${ }^{11}$ and doxetaxel. ${ }^{10}$ Targeted agents include the TKIs erlotinib ${ }^{12}$ and gefitinib, which target the epidermal growth factor receptor (EGFR). Gefitinib was removed from the North American market after a large, randomized trial, ISEL, failed to demonstrate significance when patients with chemotherapy refractory disease were randomized in the second and third-line setting. ${ }^{13}$ In a pure second-line trial, gefitinib was equal in efficacy to docetaxel, a well established second-line option, and is used in many parts of the world in this fashion. ${ }^{14}$ Gefitinib is registered in Europe for first-second- and third-line therapy in tumors expressing the EGFR mutation. ${ }^{15}$

The phrase "maintenance therapy" refers to any therapy, chemotherapy or biologic, that is administered after the initial chemotherapy regimen, and is continued or "maintained" until progression. Other terms describing this strategy include "early second line" and "consolidation". To prevent confusion and avoid linguistic problems, the phrase "maintenance therapy" will be used solely in this review.

Maintenance therapy, following first-line chemotherapy, has been previously tested in randomized trials with modest results. Based on the available data, the 2003 American Society of Clinical Oncology guidelines indicated that "first-line chemotherapy should be administered for no more than 6 cycles in patients with stage IV NSCLC", and then discontinued until progression is proven. ${ }^{16}$ The heterogeneous approach used in previous maintenance therapy trials may have impaired the interpretability of results; multiple approaches were used including continuation of the original therapy, continuation of a portion of the original doublet, or a "switch" strategy to a non-cross-resistant chemotherapy. Administering a new cross-resistant therapeutic for maintenance therapy after a first-line chemotherapy doublet may make the most biologic sense as it exposes the tumor to a novel agent.

The phrase "early versus delayed" refers to an agent that is administered after first-line chemotherapy (early) before progression, or that same agent given upon progression (delayed). It is important to understand this distinction. Mandating that treatment with the same agent given earlier (maintenance) or later (delayed) would be the truest test of the concept of maintenance therapy. The best trial design to test this concept would be a cross-over to the same 
agent upon progression in a randomized placebo controlled trial.

\section{Chemotherapy Previous trials}

One of the first studies examining maintenance therapy was carried out by Westeel et $\mathrm{al}^{2}$ and involved the use of vinorelbine. A total of 573 patients were treated with mitomycin, ifosphamide, and cisplatin chemotherapy for 4 cycles. Of the 227 patients who responded, 181 were randomized to either intravenous vinorelbine $\left(25 \mathrm{mg} / \mathrm{m}^{2}\right.$ weekly for 6 months) or no further treatment was provided. In this trial, no differences were observed for OS (HR 1.08 $P=0.48$ ) or for PFS.

Brodowicz et al showed that gemcitabine administered to NSCLC patients without progression after first-line treatment with a gemcitabine/cisplatin combination provided significant improvement in the time to progression as compared to best supportive care. ${ }^{3}$ PFS was prolonged when gemcitabine was continued, when measured either from the start date of chemotherapy or from the date of randomization $(P<0.001)$. OS was 13 months in the gemcitabine maintenance arm, versus 11 months in the best supportive care arm $(P=0.19)$. This trial did not meet statistical significance, probably because the gemcitabine that was tested in the maintenance portion was not a new therapeutic maneuver; rather it was part of the original gemcitabine/cisplatin doublet.

More recent trials have focused on using approved second-line therapies as maintenance options. In TAX 317 , patients randomized to docetaxel experienced a 3-month improvement in survival upon progression after a platinum doublet. ${ }^{10}$ Fidias et al designed a trial to determine if administering docetaxel before progression (early) or upon progression (delayed) improved patient outcomes. A total of 309 patients who completed gemcitabine plus carboplatin (GC) were randomized to early docetaxel versus delayed docetaxel. ${ }^{17}$ Median PFS for early docetaxel was 5.7 months which was significantly greater $(P=0.0001)$ than delayed docetaxel, at 2.7 months. Median OS for immediate docetaxel (12.3 months) was greater than for delayed docetaxel (9.7 months) but the difference was not statistically significant $(P=0.0853)$, as the primary endpoint of the trial was PFS. Among 153 patients randomized to immediate docetaxel, $145(95 \%)$ received at least 1 cycle, while of the patients randomized to the delayed group, only $63 \%$ received 1 cycle of docetaxel. This occurred despite mandating that patients receive docetaxel upon progression.
Reasons for why the 58 patients did not receive treatment included progression of the disease and patient decision. Additional patient review revealed that many of these patients had experienced significant symptomatic deterioration by the time they reached progressive disease and were unable to receive docetaxel therapy. These results suggest that NSCLC patients may be healthier and more likely to proceed to additional therapy if it is offered immediately after front-line therapy, before progression occurs.

\section{New data with chemotherapy}

The most recent chemotherapy to be tested in the maintenance setting is pemetrexed. ${ }^{4}$ In this trial, 663 patients with stage IIIB/lV NSCLC who had not progressed after four cycles of platinum-based chemotherapy were randomized in a 2:1 fashion to either pemetrexed $500 \mathrm{mg} / \mathrm{m}^{2}$ every 3 weeks intravenously plus best supportive care or to placebo plus best supportive care until progression, undue toxicity, or voluntary withdrawal. All patients received vitamin B12 and folic acid supplementation and dexamethasone prophylaxis. The initial chemotherapy doublet was selected by the investigator and included gemcitabine carboplatin, gemcitabine cisplatin, paclitaxel carboplatin, paclitaxel cisplatin, docetaxel carboplatin, or docetaxel cisplatin. The doublet pemetrexed platinum was not one of the permitted induction doublets as the study design was planned prior to the pemetrexed platinum registration trial. Patients had to have a performance status (ECOG) of 0 or 1, and have had a complete response, partial response, or stable disease in response to first-line chemotherapy.

Patients were stratified for ECOG performance ( 0 versus 1$)$, sex (female versus male), best response to induction therapy (complete response versus partial response versus stable disease), non-platinum component of induction therapy (gemcitabine versus paclitaxel versus docetaxel), and history of brain metastases (yes versus no). It is important to note that patients were not stratified to histology as the differential benefit of pemetrexed in non-squamous histology was not known at that time. Of the patients randomized, 73\% were men and $60 \%$ PS 1 . The majority were smokers $(73 \%$ and $71 \%$ ) in both groups. The non-squamous population was $74 \%$ in the pemetrexed group and $70 \%$ in the placebo group. This patient profile would be similar to what is seen in everyday practice. The median number of cycles was 5 (range 1-55) for pemetrexed and 3.5 (range 1-46) for the placebo. Fortyeight percent of patients in the pemetrexed group and $27 \%$ of patients in the placebo group remained on the study treatment for 6 or more cycles and $23 \%$ and $9 \%$ respectively continued 
treatment for 10 or more cycles. The treatment was well tolerated with few grade 3 or 4 toxicities (as defined by NCIC Common Toxicity Criteria) (Table 1).

PFS was significantly improved for the pemetrexed arm (HR $0.60 P<0.00001$ ). OS was also improved favoring the pemetrexed arm (HR $0.79 P=0.012)$ with a survival advantage of 2.8 months (Figure 1). In the non-squamous population, PFS (HR $0.44 P<0$. 0001) and OS (HR 0.70 $P=0.002$ ) was significantly improved. Within this select group of patients who had not progressed with non-squamous disease, the survival improved by an unprecedented 5.3 months, from 10.3 to 15.5 months (Figure 2).

After unblinding, 51\% of the pemetrexed arm and 67\% of the placebo arm went on to further therapy (Table 2). The trial has been criticized for the fact that only $18 \%$ of the placebo arm received pemetrexed. Unlike an early versus delayed trial design, patients in group B (placebo) were not mandated to receive pemetrexed on progression. This may be valid, but $29 \%$ of group B received docetaxel upon progression, which in randomized trials is equal in efficacy to pemetrexed in the second-line setting. The reduced number of patients receiving pemetrexed in the placebo arm may have also been due in part to symptomatic progression, similar to what was observed in the Fidias trial. This supports the concept of maintenance therapy.

Several researchers have shown a greater effect of pemetrexed in patients with non-squamous histology as opposed to other histological subtypes. ${ }^{5,6}$ Based on these data, the current indication for pemetrexed specifies use in the non squamous histology subgroup. While these data were not available at the time of Ciuleanu et al's trial design, an analysis of the results of patient-administered maintenance pemetrexed

Table I Pemetrexed maintenance: grades 3 and 4 toxicities

\begin{tabular}{lll}
\hline Toxicity type & Pemetrexed $\mathbf{r x \%}$ & Control rx\% \\
\hline $\begin{array}{lll}\text { Grade 3-4 treatment-related } \\
\text { toxicities }\end{array}$ & \\
Neutropenia & $3 \%$ & $<1 \%$ \\
Anemia & $3 \%$ & $<1 \%$ \\
Fatigue & $5 \%$ & $<1 \%$ \\
Anorexia & $2 \%$ & $<1 \%$ \\
Infection & $2 \%$ & 0 \\
Diarrhea & $<1 \%$ & 0 \\
Nausea & $<1 \%$ & $<1 \%$ \\
Vomiting & $<1 \%$ & 0 \\
Neuropathy & $<1 \%$ & 0 \\
Mucositis/stomatitis & $<1 \%$ & 0 \\
Rash & $<1 \%$ & 0 \\
\hline
\end{tabular}

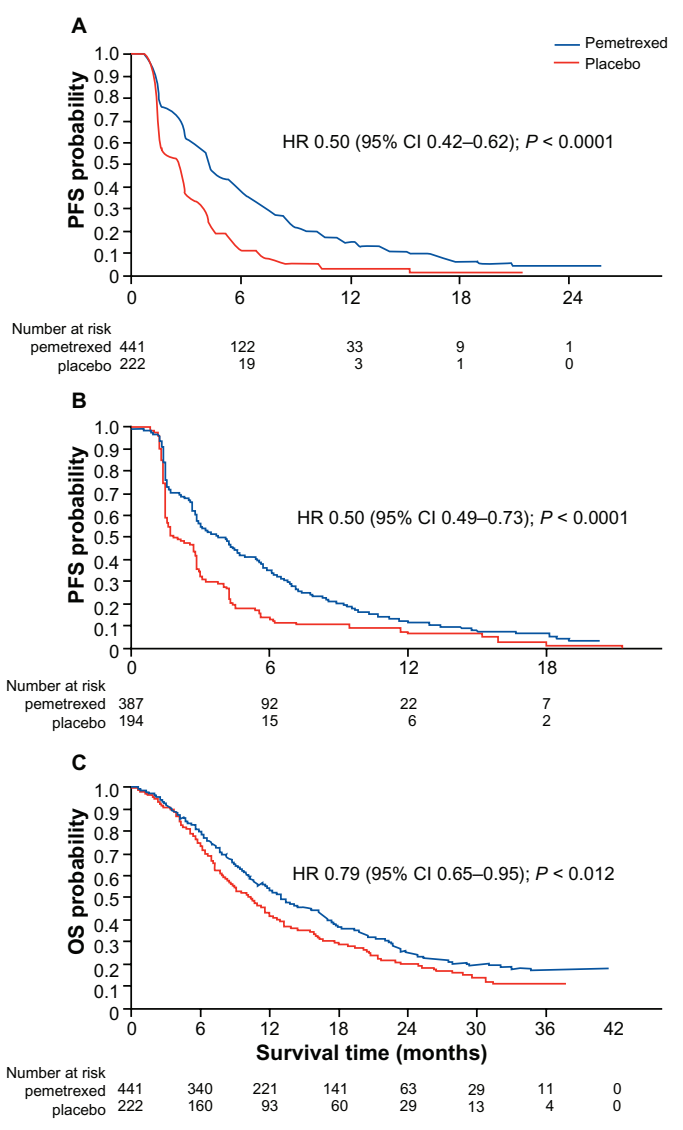

Figure I PFS (investigator assessed data $[A]$ and independent review $[B]$ and overall survival [C]). A) PFS as assessed by the investigators in the intention-to-treat population of 663 randomly assigned patients. B) PFS data from independent, central review of scans available from $58 \mathrm{I}$ randomly assigned patients. C) OS in the intention-to-treat population of 663 randomly assigned patients.

Abbreviations: PFS, progression-free survival; OS, overall survival.

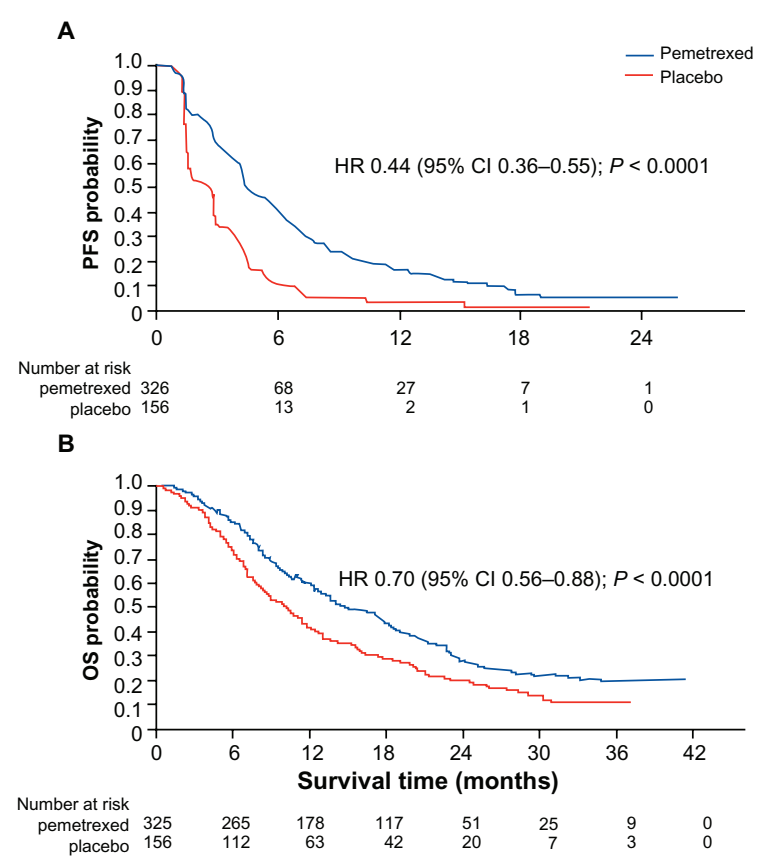

Figure 2 Pemetrexed maintenance: non-squamous subgroup, PFS and OS. Abbreviations: PFS, progression-free survival; OS, overall survival. 
Table 2 Pemetrexed maintenance: subsequent therapy

\begin{tabular}{lll}
\hline & $\begin{array}{l}\text { Pemetrexed } \\
(\mathbf{n}=44 \mathrm{I})\end{array}$ & $\begin{array}{l}\text { Placebo } \\
(\mathbf{n}=\mathbf{2 2 2})\end{array}$ \\
\hline $\begin{array}{l}\text { Any anticancer systemic } \\
\text { therapy }\end{array}$ & $227(5 \mathrm{I} \%)$ & $149(67 \%)$ \\
Pemetrexed & $4(<1 \%)$ & $4 \mathrm{I}(18 \%)$ \\
Docetaxel & $99(22 \%)$ & $65(29 \%)$ \\
Erlotinib & $95(22 \%)$ & $46(21 \%)$ \\
Gefitinib & $59(13 \%)$ & $22(10 \%)$ \\
Vinorelbine & $57(13 \%)$ & $37(17 \%)$ \\
Gemcitabine & $4 \mathrm{I}(9 \%)$ & $30(14 \%)$ \\
Carboplatin & $32(7 \%)$ & $21(9 \%)$ \\
Cisplatin & $23(5 \%)$ & $13(6 \%)$ \\
Paclitaxel & $18(4 \%)$ & $14(6 \%)$ \\
\hline
\end{tabular}

Notes: Data are number (\%) of patients who received post-discontinuation therapy.

Patients could have received more than one additional line of therapy.

confirms that the benefit was accrued predominantly by the non-squamous group.

\section{Meta-analysis for maintenance chemotherapy}

Maintenance and duration of chemotherapy for advanced NSCLC was systematically reviewed in a metaanalysis of 13 randomized trials of 3,027 patients. ${ }^{9}$ Extending chemotherapy improved PFS substantially (HR 0.75 $(P<0.00001))$, and OS was marginally improved as well (HR $0.92(P=0.03))$. Subgroup analysis revealed that the effects on PFS were greater for trials extending chemo-therapy with third-generation or current regimens, than with older regimens (HR $0.70(P=0.003)$ ). This meta-analysis included the pemetrexed maintenance trial by Ciuleanu et al, however the OS data were preliminary at that time.

\section{Biologics \\ Previous trials}

Biologic and targeted therapy has improved survival in many solid tumor settings. Their side effect profile differs from chemotherapy and generally allows for their use in a continued fashion. Both VEGF and EGFR targets have been tested. The monoclonal antibody bevacizumab inhibits angiogenesis, as proven in phase 111 randomized placebo controlled trials. The agents inhibiting the EGFR include the monoclonal antibody cetuximab and oral TKIs, erlotinib, and gefitinib.

In the Eastern Cooperative Group Trial E4599, patients with advanced stage NSCLC were randomized to a carboplatin/paclitaxel doublet for 6 cycles with bevacizumab or placebo. ${ }^{18}$ Bevacizumab or placebo was administered with the initial chemotherapy and continued in a maintenance fashion, until progression. This trial was positive for its primary endpoint of OS; patients treated with bevacizumab in their initial chemotherapy had a median survival of 12.3 months versus 10.3 months without bevacizumab (HR 0.79, CI 0.67-0.092, $P<0.003$ ). However, it is unknown if bevacizumab needs to be administered with the initial chemotherapy or just afterwards as maintenance, as the trial design did not address this question. Similarly, in the AVAIL trial, bevacizumab at $7.5 \mathrm{mg} / \mathrm{kg}$ or $15 \mathrm{mg} / \mathrm{kg}$ was given with cisplatin/gemcitabine. ${ }^{19}$ This trial was positive for the primary endpoint of PFS. The HR for bevacizumab for both doses, $7.5 \mathrm{mg} / \mathrm{kg}$ (HR 0.75 $P<0.0026)$ and $15 \mathrm{mg} / \mathrm{kg}(\mathrm{HR} 0.82 P<0.031)$ were significantly superior to placebo. A secondary endpoint of OS was similar between placebo (13.1 months) and bevacizumab $7.5 \mathrm{mg} / \mathrm{kg}$ (13.6 months) or $15 \mathrm{mg} / \mathrm{kg}$ (13.4 months). More than two thirds of patients in the AVAIL trial received further therapy when they reached progression. As more lines of therapy are now administered to patients with metastatic NSCLC, the survival advantage conferred by each therapeutic becomes more difficult to determine. Like the previous E4599 trial, the randomization in AVAIL occurred initially before chemotherapy was initiated so it is unknown if bevacizumab is best administered concurrent with chemotherapy, after chemotherapy in the maintenance fashion, or if both options are equally optimal. Bevacizumab is approved for first-line treatment for advanced NSCLC with chemotherapy, and can be administered until disease progression.

Phase 111 trials have studied the monoclonal antibody cetuximab, and have examined EGFR as a target. BMS 099 found no difference in PFS when cetuximab was added to a taxane-carboplatin doublet. ${ }^{20}$ The FLEX trial examined the addition of the monoclonal antibody cetuximab to first-line chemotherapy doublet cisplatin/vinorelbine. ${ }^{21}$ Cetuximab or placebo was given with initiation of four cycles of the doublet, and then continued until disease progression. Similar to the bevacizumab trials, the trial design dictated that cetuximab or placebo was given in a maintenance fashion following chemotherapy. This positive trial demonstrated a 1-month improvement in OS. Like E4599 and AVAIL, it is unclear if the benefit observed was due to the administration of maintenance cetuximab or because of synergy with chemotherapy.

INTACT 1 and INTACT 2 examined the addition of gefitinib to first-line chemotherapy and the continuation of gefitinib after chemotherapy was completed, and showed 
that there was no additional benefit to survival. ${ }^{22,23}$ Similarly, TALENT and TRIBUTE similarly examined the influence of erlotinib, but like gefitinib, the addition of erlotinib to first-line platinum doublet chemotherapy did not significantly prolong survival. ${ }^{24,25}$ However, analyses suggested a beneficial effect when erlotinib was administered for maintenance therapy after chemotherapy was completed. In the TALENT study, patients who received erlotinib for more than 150 days had a significantly increased duration of response versus those receiving a placebo. In the TRIBUTE study, patients who survived beyond completion of chemotherapy and were treated with erlotinib had a median OS of 15.4 months, which was significantly longer than the 13.8 months reported in the placebo group. This was the first indication that erlotinib may be beneficial when administered as a maintenance therapy.

\section{New data biologics}

New data are emerging for erlotinib maintenance in both the SATURN and ATLAS trials. Patients enrolled in SATURN were treated with a platinum doublet of the investigator's choice (cisplatin/paclitaxel; cisplatin/ gemcitabine; cisplatin/docetaxel cisplatin/vinorelbine; carboplatin/gemcitabine; carboplatin/docetaxel carboplatin/ paclitaxel). ${ }^{7}$ Almost 2000 patients received first-line therapy, of which 889 nonprogressing patients were randomized to erlotinib $150 \mathrm{mg}$ daily or placebo. About $60 \%$ of the nonprogressing patients had achieved stable disease after their chemotherapy regimen, and most of the remaining patients had achieved a partial response ( $<1 \%$ complete response). Approximately 45\% had an adenocarcinoma and $40 \%$ had a squamous histology, and all patients had a performance status of 0 or 1 .

Both coprimary endpoints, PFS in all patients and PFS in patients with EGFR IHC+ tumors, were positive. The PFS $\mathrm{HR}$ in the intention-to-treat population was 0.71 (95\% CI, $0.62-0.82 ; P<0.0001)$, representing an improvement of $41 \%$ ( $29 \%$ risk reduction) over a follow-up period of up to 88 weeks. At 24 weeks, the rate of PFS was nearly double in the erlotinib group as compared to placebo (31\% versus $17 \%$ ). The coprimary endpoint assessed a subgroup of patients with tumors positive for EGFR protein expression, as determined by immunohistochemistry, a key target of erlotinib. In this EGFR positive group, the relative improvement in PFS was $31 \%$ (HR 0.69; 95\% CI, 0.58-0.92; $P<0.0001$ ). At 24 weeks, the proportion of patients who had not progressed was $32 \%$ versus 18\%, favoring erlotinib (Figure 3 ). Adverse effects in these patients were comparable to those well described in previous erlotinib clinical trials, and included rash and diarrhea. There were only 5 withdrawals for any adverse event in the erlotinib group as compared to two withdrawals in the placebo group (Table 3). There was an OS benefit of 1 month: 12 months versus 11 months $(\mathrm{HR}=0.81$ $\log$-rank $P=0.0088$ ). In the non-squamous group, OS was greater than three months: 13.7 versus 10.5 (HR 0.79 logrank $P=0.0194)$. The OS and clinical benefit was consistent across subgroups, irrespective of histology, race, or smoking status. Erlotinib treatment was very well tolerated with a low discontinuation rate compared to placebo. Follow-up data indicate that in the erlotinib group, $71 \%$ of patients went on to receive additional therapy for their disease, similar to those in the placebo group, $72 \%$ (Table 4). Biomarker results for EGFR mutation in SATURN showed a marked benefit in progression free survival (HR $0.01(0.04-0.25) P<0.0001)$. However, the benefit was seen in patients with the wild-type mutation as well (HR $0.78(0.63-0.96) P=0.018)$. This was similar to the HR for the whole group regardless of the EGFR mutation status (HR $0.71(0.62-0.82) P<0.001)$ indicating
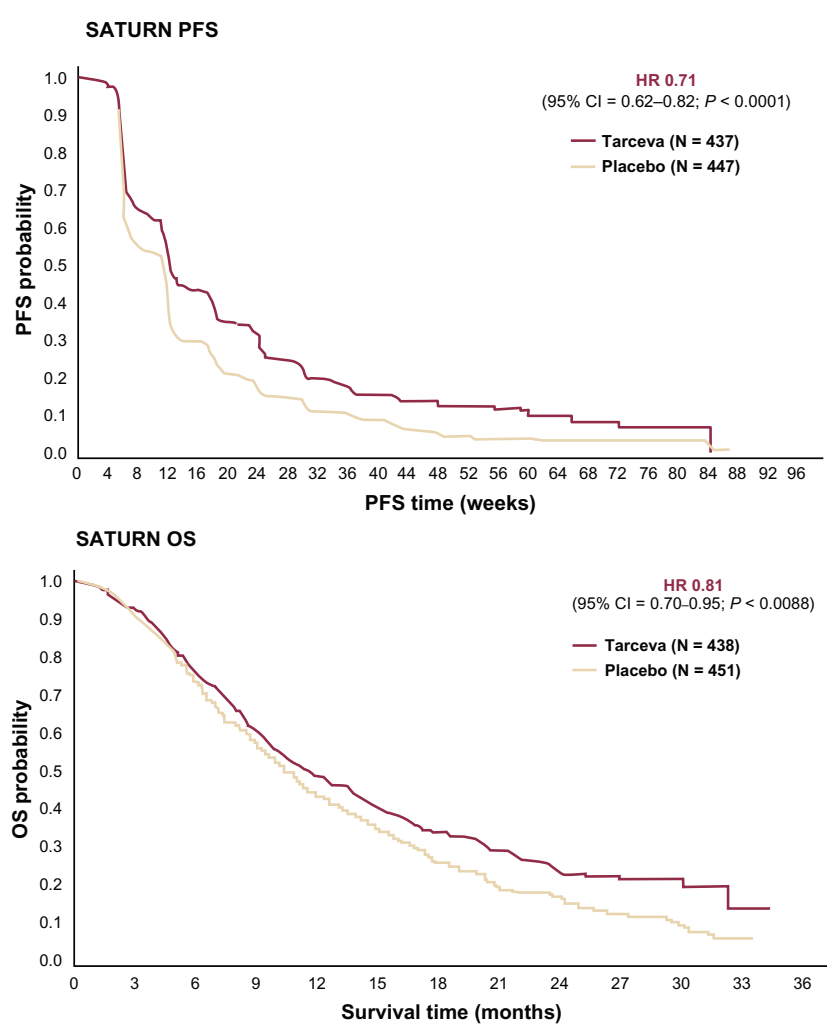

Figure 3 Erlotinib maintenance: PFS and OS.

Abbreviations: PFS, progression-free survival; OS, overall survival. 
Table 3 Grades 3 and 4 adverse events (AEs) from SATURN

\begin{tabular}{lll}
\hline AEs occurring in \\
$\geq 1$ I $\%$ of patients & SATURN trial & \\
\cline { 2 - 3 } & Erlotinib $(n=433)$ & Placebo $(n=\mathbf{4 4 5})$ \\
\hline Rash $(\%)$ & 60 & 9 \\
Grade 3 & 9 & 0 \\
Grade 4 & 0 & 0 \\
Diarrhea $(\%)$ & 20 & 4 \\
Grade 3 & 2 & 0 \\
Grade 4 & 0 & 0 \\
\hline
\end{tabular}

that the need to test for EGFR status prior to commencing therapy is less clear.

The ATLAS study evaluated a combination maintenance therapy of the EGFR TKI erlotinib with the VEGF inhibitor bevacizumab in stage IIIb/IV patients who had not progressed after being treated with bevacizumab plus a platinum-containing doublet therapy. ${ }^{8}$ A total of 768 participants were randomized to bevacizumab $\left(15 \mathrm{mg} / \mathrm{m}^{2}\right.$ administered every 3 weeks) plus erlotinib or same-schedule bevacizumab plus placebo. The primary objective was PFS, and the trial was discontinued by the data safety monitoring committee at the second interim evaluation because this primary endpoint was met. The improvement in PFS was approximately 1 month, 4.8 months versus 3.7 months, (HR 0.72; 95\% CI, 0.59-0.81; $P=0.0012$ ) which represents a $28 \%$ reduction in progression. The safety profile was acceptable (Table 5). The improvement in PFS was seen across multiple subgroups, including those defined by gender, histology, age, and smoking status. OS data are pending. The percentage of patients who received subsequent therapy was over $50 \%$ in both arms (Table 6). A confounding factor in the ATLAS trial, influencing the interpretation of results, is that patients in the control arm (bevacizumab and placebo) could receive the experimental

Table 4 Subsequent therapies from SATURN trial

\begin{tabular}{|c|c|c|}
\hline & \multicolumn{2}{|c|}{$\begin{array}{l}\text { Patients with at least one subsequent } \\
\text { therapy (\%) }\end{array}$} \\
\hline & Erlotinib $(n=438)$ & Placebo $(n=45 I)$ \\
\hline All classes & 7I & 72 \\
\hline $\begin{array}{l}\text { Taxanes (including } \\
\text { docetaxel) }\end{array}$ & 30 & 31 \\
\hline $\begin{array}{l}\text { Antimetabolites } \\
\text { (including pemetrexed) }\end{array}$ & 24 & 23 \\
\hline Antineoplastic agents & 16 & 18 \\
\hline $\begin{array}{l}\text { Tyrosine-kinase } \\
\text { inhibitors }\end{array}$ & II & 21 \\
\hline Platinum compounds & 9 & 12 \\
\hline
\end{tabular}

combination therapy (bevacizumab and erlotinib) on disease progression. The high amounts of bevacizumab and erlotinib administered as subsequent treatment may have influenced the OS results.

Publication of SATURN and ATLAS is awaited. Erlotinib has not yet been registered by the FDA for use as maintenance therapy. The final results of the biomarker data may shed light on who will most benefit from this strategy.

\section{Conclusion}

Maintenance therapy in NSCLC refers to the introduction of a therapeutic agent after first-line chemotherapy is completed and before disease progression occurs. Ideally in a clinical trial, the maintenance therapy being tested would be randomized with a placebo, and mandated to be used in the placebo group upon disease progression. PFS is an important endpoint, but is weakened as a goal in a maintenance trial; if a therapeutic agent is given before progression, in contrast to administering upon progression, one might expect that PFS would be prolonged. The true test that maintenance therapy may be useful and clinically meaningful to the patient is an improvement in OS. Thus, the two strategies necessary for an ideal maintenance trial include: (1) the same therapeutic agent administered in maintenance or on disease progression; and (2) the primary endpoint would be a statistical significant improvement in OS. Finally, as we are asking our patients to continue treatment without a break, the therapy used in maintenance should be well tolerated and not lead to a decrease in quality of life.

Recent trials with maintenance chemotherapy have met the goal of improved OS. Pemetrexed and erlotinib appear to provide the most benefit and are also active in a secondline setting. Docetaxel also prolongs survival when used as a second-line therapy. ${ }^{10}$ Docetaxel was tested in a maintenance fashion as early versus delayed therapy, and the primary endpoint, PFS, was improved even though only $70 \%$ of the patients that were randomized to the "delayed" arm received docetaxel. This was regardless of the fact these patients were monitored in a clinical trial setting. ${ }^{16}$ Using an approved second-line therapy for maintenance ensures fewer patients are lost to either follow up, death, or deterioration, meaning that they are unable to receive further treatment.

Pemetrexed was tested in a maintenance fashion. ${ }^{4}$ A marked benefit of pemetrexed maintenance therapy was observed as compared to placebo; an improvement of 5.3 months was observed in non squamous histology 
Table 5 Grade 3 and 4 adverse events (AEs) from ATLAS

\begin{tabular}{lll}
\hline & $\begin{array}{l}\text { Bev + Erlotinib, } \\
\mathbf{n}(\%)(\mathbf{n = 3 6 7 )}\end{array}$ & $\begin{array}{l}\text { Bev + Placebo, } \\
\mathbf{n}(\%)(\mathbf{n}=\mathbf{3 6 8})\end{array}$ \\
\hline Any grade AE & $349(95.1 \%)$ & $313(85.1 \%)$ \\
Grade 3-4 AE & $162(44.1 \%)$ & $112(30.4 \%)$ \\
Any grade AE & $349(95.1 \%)$ & $313(85.1 \%)$ \\
Grade 3-4 events & & \\
of interest & & $2(0.5 \%)$ \\
Rash & $38(10.4 \%)$ & $3(0.8 \%)$ \\
Diarrhea & $34(9.3 \%)$ & $17(4.6 \%)$ \\
Infection & $15(4.1 \%)$ & $5(1.4 \%)$ \\
Hemorrhage & $6(1.6 \%)$ & $2(0.5 \%)$ \\
Pulmonary & $3(0.8 \%)$ & 0 \\
hemorrhage & & $4(1.1 \%)$ \\
GI perforation & $2(0.5 \%)$ & $1(0.3 \%)$ \\
Neutropenia & $2(0.5 \%)$ & $7(1.9 \%)$ \\
Thrombocytopenia & $2(0.5 \%)$ & $21(5.7 \%)$ \\
Proteinuria & $6(1.6 \%)$ & $5(1.4 \%)$ \\
Hypertension & $20(5.4 \%)$ & $10(2.7 \%)$ \\
ATE & $8(2.2 \%)$ & $2(0.5 \%)$ \\
VTE & $4(1.1 \%)$ & \\
Wound healing & $2(0.5 \%)$ & \\
complications & &
\end{tabular}

Abbreviations: ATE, arterial thrombotic event; VTE, verous thrombotic event.

patients with good performance status and disease control after first-line doublet therapy. Pemetrexed is efficacious in promoting survival when used as second-line therapy upon disease progression. ${ }^{11}$ The median number of pemetrexed cycles with maintenance was 5 , similar to the number in a second-line setting, in which the median was 4 cycles; this result once again suggests that administering pemetrexed

Table 6 Subsequent therapies from ATLAS trial

\begin{tabular}{lll}
\hline & $\begin{array}{l}\text { Bev + placebo, } \\
\mathbf{n}(\%)(\mathbf{n}=373)\end{array}$ & $\begin{array}{l}\text { Bev + erlotinib, } \\
\mathbf{n}(\%)(\mathbf{n}=\mathbf{3 7 0})\end{array}$ \\
\hline $\begin{array}{l}\text { Patients who received } \\
\text { subsequent therapy }\end{array}$ & $207(55.5 \%)$ & $186(50.3 \%)$ \\
$\begin{array}{l}\text { Anti-VEGF } \\
\text { (bevacizumab) }\end{array}$ & $149(39.9 \%)$ & $92(24.9 \%)$ \\
$\begin{array}{l}\text { EGFR-targeted } \\
\text { (erlotinib) }\end{array}$ & $148(39.7 \%)$ & $147(39.7 \%)$ \\
$\begin{array}{l}\text { Chemotherapy } \\
\text { Radiotherapy }\end{array}$ & $106(28.4 \%)$ & $123(33.2 \%)$ \\
$\begin{array}{l}\text { Investigational therapy } \\
\text { Surgery/procedure }\end{array}$ & $35(9.4 \%)$ & $25(6.8 \%)$ \\
\hline
\end{tabular}

*Pemetrexed was most common chemotherapy.

Abbreviations: EGFR, epidermal growth factor receptor; VEGF, vascular endothelial growth factor. earlier, as a maintenance therapy, is beneficial for patient survival. Although this trial has been criticized for not mandating that patients on the placebo arm receive pemetrexed, an "early versus delayed" design was not used. The actual percentage of patients in this group who received subsequent therapy was higher in the placebo arm than the maintenance pemetrexed arm, and many patients in the placebo arm received docetaxel which is known to have equal efficacy to pemetrexed. This is a positive trial and pemetrexed is approved to be used in this fashion by regulatory agencies in North America. Application of maintenance pemetrexed must be evidence based. Patients with advanced non-squamous histology who have not progressed after four cycles of a platinum doublet not containing pemetrexed would meet inclusion criteria for pemetrexed maintenance therapy.

Erlotinib was tested in a maintenance fashion in both SATURN and ATLAS. ${ }^{7,8}$ The difference between these trials is that ATLAS included bevacizumab with erlotinib as a maintenance therapy. The bevacizumab was part of the induction treatment, similar to E4599 and AVAIL..$^{18,19}$ Both SATURN and ATLAS demonstrated similar results; both are positive for PFS with similar HRs of 0.71 for SATURN and 0.72 for ATLAS. Most impressive is the OS advantage for the SATURN trial, with an HR of 0.81. We await publication of both trials.

Neither the pemetrexed or erlotinib maintenance trials were perfectly designed. Neither trial employed a design where patients on the placebo arm were mandated to cross over to the drug being tested on disease progression. Nonetheless, the majority of patients in both trials received subsequent therapies. Both trials reached statistical significance in prolonging OS, in spite of multiple therapies used to patients with metastatic NSCLC.

Who is the winner? The benefit to OS is similar with either pemetrexed or erlotinib, with HRs of 0.79 and 0.81 respectively. Cross-trial comparisons should be discouraged. Both therapies have their advantages and disadvantages. Not only do the side effect profiles of pemetrexed and erlotinib differ, but methods of drug administration vary as well. Pemetrexed is an intravenous drug that requires prophylactic dexamethasone, vitamin B12 injections, and daily folic acid supplementation. Side effects are uncommon, but may include fatigue, nausea, and vomiting. In contrast, erlotinib is an oral drug that patients can take at home. Although rashes occur in the majority of patients, severe grade 3 rashes (as defined by NCIC Common Toxicity Criteria) or other side effects are uncommon. To experienced oncologists, both therapies 
can be given to similar patient groups. The ultimate decision should be made with the individual patient in mind, and in the case of pemetrexed, the patient's tumor histology.

To ask our patients to take maintenance therapy requires careful discussion with them. Many patients may choose to have a drug holiday after 3 or 4 months of a platinum regimen. Residual nausea, fatigue, and alopecia can take time to resolve. Many may refuse maintenance therapy as it requires monitoring visits in addition to treatment.

In conclusion, the standard of care in metastatic NSCLC has been to treat patients with a platinum doublet for four to six cycles and, upon progression, offer second-line therapy. Proven second-line options include docetaxel, pemetrexed, and erlotinib. Trials have demonstrated a trend to OS improvement with docetaxel maintenance therapy; pemetrexed and erlotinib maintenance therapies have also been found to improve OS. The strategy of maintenance therapy competes with the strategy of second-line or treatment holiday; however, it has the advantage of allowing more patients to receive treatment in contrast to waiting until a patient's disease progresses. This may mean that $30 \%$ more patients will receive treatment. Administering approved second-line therapies early, before the disease progresses, has shown to be a worthwhile maneuver to prolong patients' survival. The strategy of administering upfront maintenance therapy after first-line chemotherapy with either pemetrexed or erlotinib provides an excellent treatment option for a subgroup of NSCLC patients; this approach improves PFS and OS in a clinically significant way and represents a paradigm shift in the management of this disease.

\section{Disclosure}

The author discloses no conflicts of interest.

\section{References}

1. Chiller JH, Harrington D, Belani CP, et al. Comparison of four chemotherapy regimens for advanced non-small-cell lung cancer. NEngl J Med. 2002;346:92-98.

2. Westeel V, Quoix E, Moro-Sibilot D, et al. Randomized study of maintenance vinorelbine in responders with advanced non-small-cell lung cancer. J Natl Cancer Inst. 2005;97:499-506.

3. Brodowicz T, Krzakowski M, Zwitter M, et al. Cisplatin and gemcitabine first-line chemotherapy followed by maintenance gemcitabine or best supportive care in advanced non-small cell lung cancer: A phase III trial. Lung Cancer. 2006;52:155-163.

4. Ciuleanu T, Brodowicz T, Zielinski C, et al. Maintenance pemetrexed plus best supportive care versus placebo plus best supportive care for non-small-cell lung cancer: a randomised, double-blind, phase 3 study. Lancet. 2009;374:1432-1440.

5. Scagliotti GV, Parikh P, von Pawel J, et al. Phase III study comparing cisplatin plus gemcitabine with cisplatin plus pemetrexed in chemotherapy-naive patients with advanced-stage non-small-cell lung cancer. J Clin Oncol. 2008;26:3543-3551.
6. Peterson P, Park K, Fossella F, et al. Is pemetrexed more effective in patients with non-squamous histology? A retrospective analysis of a phase III trial of pemetrexed versus docetaxel in previously treated patients with advanced non-small cell lung cancer (NSCLC). Eur J Cancer. 2007;5:363-364.

7. Cappuzzo F, Ciuleanu T, Stelmakh L, et al. SATURN: A double-blind, randomized, phase III study of maintenance erlotinib versus placebo following nonprogression with first-line platinum-based chemotherapy in patients with advanced NSCLC. ASCO Meeting Abstracts. 2009;27:8001.

8. Miller VA, O'Connor P, Soh S, et al. A randomized, double-blind, placebo-controlled, phase IIIb trial (ATLAS) comparing bevacizumab (B) therapy with or without erlotinib (E) after completion of chemotherapy with B for first-line treatment of locally advanced, recurrent, or metastatic non-small cell lung cancer (NSCLC). J Clin Oncol. 2009;27:(18s):abstr LBA8002.

9. Soon YY, Stockler MR, Askie LM, et al. Duration of chemotherapy for advanced non-small-cell lung cancer: A systematic review and metaanalysis of randomized trials. J Clin Oncol. 2009;27:3277-3283.

10. Shepherd FA, Dancey J, Ramlau R, et al. Prospective randomized trial of docetaxel versus best supportive care in patients with non-small-cell lung cancer previously treated with platinum-based chemotherapy. J Clin Oncol. 2000;18:2095-2103.

11. Hanna N, Shepherd FA, Fossella FV, et al. Randomized phase III trial of pemetrexed versus docetaxel in patients with non-small-cell lung cancer previously treated with chemotherapy. J Clin Oncol. 2004;22:1589-1597.

12. Shepherd FA, Pereira J, Ciuleanu TE, et al. Erlotinib in previously treated non-small-cell lung cancer. N Engl J Med. 2005;353:123-132.

13. Thatcher N, Chang A, Parikh P, et al. Gefitinib plus best supportive care in previously treated patients with refractory advanced non-smallcell lung cancer: Results from a randomized, placebo-controlled, multicentre study (Iressa Survival Evaluation in Lung Cancer). Lancet. 2005;366:1527-1537.

14. Kim ES, Hirsh V, Mok T, et al. Gefitinib versus docetaxel in previously treated non-small-cell lung cancer (INTEREST): a randomised phase III trial. Lancet. 2008;372:1809-1818.

15. AstraZeneca press release, April 23, 2009. IRESSA (Gefitinib) recommended for approval for the treatment of non-small cell lung cancer in Europe. Available at: http://www.astrazeneca.com/media/ latest-press-releases/2009/iressa-chmp?itemId=5585247 Accessed 2010 March 10.

16. Pfister DG, Johnson DH, Azzoli CG, et al. American society of clinical oncology treatment of unresectable non-small-cell lung cancer guideline: update. J Clin Oncol. 2003;22:330-353.

17. Fidias PM, Dakhil SR, Lyss AP, et al. Phase III study of immediate compared with delayed docetaxel after front-line therapy with gemcitabine plus carboplatin in advanced non-small-cell lung cancer. J Clin Oncol. 2009;27:591-598.

18. Sandler AB, Gray R, Perry MC, et al. Paclitaxel-carboplatin alone or with bevacizumab for non-small cell lung cancer. $N$ Engl J Med. 2006;355:2542-2550.

19. Reck M. von Pawel J, Zatloukal P, et al. Phase III trial of cisplatin plus gemcitabine with either placebo or bevacizumab as first-line therapy for nonsquamous non-small cell lung cancer: AVAiL. J Clin Oncol. 2009;27:1227-1234.

20. Khambata-Ford S, Harbison CT, Hart LL, et al. Analysis of potential predictive markers of cetuximab benefit in BMS099, a phase III study of cetuximab and first-line taxane/carboplatin in advanced non-smallcell lung cancer. J Clin Oncol. 2010;28:918-27.

21. Pirker R, Szczesna A, von Pawel J, et al. FLEX: a randomized, multicenter, phase III study of cetuximab in combination with cisplatin/vinorelbine (CV) versus $\mathrm{CV}$ alone in the first-line treatment of patients with advanced non-small cell lung cancer (NSCLC). J Clin Oncol. 2008;26:1006.

22. Giaccone G, Herbst RS, Manegold C, et al. Gefitinib in combination with gemcitabine and cisplatin in advanced non-small-cell lung cancer: a phase III trial - INTACT 1. J Clin Oncol. 2004;22:777-784. 
23. Herbst RS, Giaccone G, Schiller JH, et al. Gefitinib in combination with paclitaxel and carboplatin in advanced non-small-cell lung cancer: a phase III trial - INTACT 2. J Clin Oncol. 2004;22:785-794.

24. Gatzemeier U, Pluzanska A, Szczesna A, et al. Phase III study of erlotinib in combination with cisplatin and gemcitabine in advanced non-small-cell lung cancer: the Tarceva Lung Cancer Investigation Trial. J Clin Oncol. 2007;25:1545-1552.
25. Herbst RS, Prager D, Hermann R, et al. TRIBUTE: A phase III trial of erlotinib hydrochloride (OSI-774) combined with carboplatin and paclitaxel chemotherapy in advanced non-small-cell lung cancer. J Clin Oncol. 2005;23:5892-5899.

\section{Publish your work in this journal}

Lung Cancer: Targets and Therapy is an international, peer-reviewed, open access journal focusing on lung cancer research, identification of therapeutic targets and the optimal use of preventative and integrated treatment interventions to achieve improved outcomes, enhanced survival and quality of life for the cancer patient. Specific topics covered in the journal include:
Epidemiology, detection and screening; Cellular research and biomarkers; Identification of biotargets and agents with novel mechanisms of action; Optimal clinical use of existing anticancer agents, including combination therapies; Radiation and surgery; Palliative care; Patient adherence, quality of life, satisfaction; Health economic evaluations.

Submit your manuscript here: http://www.dovepress.com/lung-cancer-targets--therapy-journal 